\title{
Marco teórico y metodológico para el análisis del movimiento social afrodescendiente
}

\section{Theoretical and methodological framework for analysis of the social movement of African descent}

\author{
JHON AnTón SÁNCHEZ* \\ RESUMEN
}

En este ensayo intento una lectura sobre elementos conceptuales y metodológicos que permitan comprender la naturaleza "del proceso organizativo afrodescendiente", dentro de dos paradigmas sobre los movimientos sociales: primero la escuela norteamericana que analiza a los movimientos sociales desde la teoría de la movilización de recursos (TRM) y los privilegia como un conjunto de actores estratégicos que se mueven en función de recursos e intereses. En segundo lugar se distingue la escuela europea, que bajo la teoría de los Nuevos Movimientos Sociales (NMS) analiza el fenómeno desde las identidades colectivas como motores que impulsan la acción de los actores dentro de un contexto cultural más complejo y sostenido en el tiempo.

Palabras clave: Movimientos sociales; Acciones colectivas; Actor; Organizaciones; Acción racional; Nuevos movimientos sociales; Grupos de interés; Identidad; Recursos.

\section{ABSTRACT}

In an attempt reading this trial on methodological and conceptual elements to understand the nature "of the organizational process of African descent, in two paradigms on social movements: the first American school that analyzes the social movements since the theory of resource mobilization (TRM) and privileges as a set of strategic players that move in terms of resources and interests. Secondly distinguishes the European school, which under the theory of the New SocialMovements (NMS) examines the phenomenon from the collective identities as the engines that drive the action of the actors within a cultural context more complex and sustained over time.

Keywords: Social movements; Collective; Actor; Organizations; Action rational; New social movements; Interest groups; Identity; Resources.

\section{PRESENTACIÓN}

Intentar anotaciones metodológicas y conceptuales sobre el "movimiento social afrodescendiente" implica varias dificultades. La más com-
* Candidato a Ph D en Antropología Política, FLACSO, sede Ecuador

Recibido: Julio 4, 2007

Aceptado: Febrero 20, 2008 
que permitan comprender el fenómeno desde su propia naturaleza.

En este ensayo se pretende hacer un esbozo conceptual sobre la teoría de los movimientos sociales. Este esfuerzo es necesario antes de aventurarnos a la comprensión del movimiento social afrodescendiente. Tal esbozo se hará en tres aspectos: a) una aproximación a la definición; b) una exploración a sus principales enfoques conceptuales; y c) una pretensión prudente de categorías, que desde el punto de vista metodológico ayudarán a descomponer el problema.

\section{¿CÓMO DEFINIR UN MOVIMIENTO SOCIAL?}

Desde Jorge León (1997:29) la categoría «movimiento social" caracteriza procesos sociales distintos de aquellos que se ajustaban al clásico concepto marxista de "luchas de clases". Se trata de una definición acuñada para entender los conflictos en las sociedades modernas. Anteriormente sobre esta categoria pesaba una definición descriptiva que trataba de determinar a grupos de personas que motivados por el cambio social se movilizaban, identificaban un oponente y generaban acciones colectivas con frecuencia difusas. Se buscaba una idea que diera cuenta de la manera cómo la gente se organizaba, cómo se integraba bajo una identidad y un fin.

Para León un movimiento social no se resume en una organización, ni una protesta, ni un programa definido. Más bien se trata de la convergencia de ideas, acciones y personas que se encaminan hacia una transformación de sus situaciones o de las instituciones definidas (1997:30). Incluso un movimiento social puede implicar modos de comportamiento, maneras de pensar, de relación entre actores que proponen acciones concretas de cambio. Un movimiento social puede o no ser expresiones de acciones colectivas contundentes (manifestaciones, protestas, paros, revueltas, tomas, levantamientos, derrocamientos), o alcanzar varios actores (organizaciones, instituciones, sectores sociales, activistas, líderes carismáticos), y a su vez tener catalizadores (organizaciones no gubernamentales, la prensa, las iglesias). Pero siempre su acción se orientaría en transformaciones o de cambio social. Un movimiento social es un concepto que identifica un proceso social, mediante el cual una sociedad cambia uno o varios aspectos de su vida o de sus instituciones (León, 1994: 30).

De modo que al intentar definir un movimiento social habria que buscar una característica clave: el interés de cambio que unos actores proponen en su contexto social. Por ejemplo, ideas de luchar por la libertad, la igualdad o la erradicación del racismo enarboladas por las organizaciones negras en el sur de Estados Unidos que intentaron transformar el orden estatuido de la segregación racial, son claras expresiones de movimiento social. Pues tal como lo determina Orlando Fals Borda (1989), los movimientos sociales son la posibilidad de estatuir un nuevo orden social.

Sin embargo, para que la sociología pueda construir adecuadamente la categoría de "movimiento social" (MS) es necesaria una lectura en doble perspectiva: las situaciones diacrónicas y sincrónicas que lo envuelven (Mouriaux y Beroud, 2000). La perspectiva diacrónica entiende al MS como un proceso de carácter no programático, lineal y uniforme. Lo observa como un fenómeno no regular que se desenvuelve en un periodo de larga duración. Comprender al MS como un proceso diacrónico implica que éste posea etapas, las cuales tienen extensión propia, y estructura particular. Se trata de una periodización de rupturas y continuidades, de singularidades en sus partes y con ritmos propios. En el caso afrodescendiente, por ejemplo, las acciones colectivas que las organizaciones realizan por subvertir el sistema al plantearse "no al racismo" o por "una sociedad sin discriminación", se pueden observar dentro de un período histórico de cambio social. Se trata de una lógica de procesos de larga duración que arranca, incluso, desde las luchas cimarronas por la libertad, pasando por acciones por la abolición de la esclavitud y la conquista de los derechos ciudadanos, y terminando con la lucha contra la pobreza, el racismo estructural y la exclusión económica, social y política (Antón 2004; Agudelo 2006).

La segunda perspectiva analítica de un movimiento social sería su carácter sincrónico. Es decir, la expresión coyuntural de una colectividad social en movimiento. Se trata del retrato en el tiempo presente y en el espacio actual de las formas de acción, de las oportunidades politicas, de las estrategias y del entorno social en que los actores se mueven. En definitiva, es el análisis del contexto del MS en sus dimensiones simbólicas, culturales, políticas, económicas y globales. 


\section{Bioetnia Volumen 5 No 1 (enero-junio), 2008}

Además de la perspectiva analítica diacrónica y sincrónica, Orlando Fals Borda (1989) identifica dos características adicionales necesarias en el análisis de los movimientos sociales: los aspectos microestructurales y macroestructurales. Lo micro encierra la vida cotidiana de un MS, es el contexto coyuntural en que los actores movilizados se encuentran. Aquí observamos manifestaciones espontáneas que van desde la protesta, hasta los mítines, tomas y levantamientos. Son acciones colectivas que pretenden alimentar la identidad del movimiento, sea ésta ecológica, ambiental, estudiantil, étnica, de opción sexual o de cualquier otra índole. Estas acciones micro estructurales en determinadas circunstancias desembocan en acciones politicas de mayor alcance o en acciones de nivel macro estructural. Es decir, los MS pasan de una reivindicación concreta a un llamado de cambio social. Así terminan proponiendo factores que incitan a la toma de medidas que conduzcan a una nueva cultura política, a una acción de cambio a las estructuras sociales. Se pasa así de lo micro a lo macro, de la protesta callejera a la propuesta, se rompe la perspectiva coyuntural y local. Además se establecen canales de doble vía, uno hacia abajo que mira reivindicaciones puntuales de derechos sociales y otro hacia arriba que pretende la transformación de la sociedad.

Esta precisión de lo micro y de lo macro en los movimientos sociales es clave para entender la dinámica de los nuevos movimientos sociales afrodescendientes en América Latina. De acuerdo con Arturo Escobar (1997), estos no sólo se concretan en el escenario de las demandas locales y particulares, o demandas de políticas culturales, sino que tales acciones de manera estructural buscan subvertir el sistema imperante, pretendiendo en últimas transformar la cultura política en que la sociedad se envuelve. Desde Escobar los MS se sitúan en un doble contexto de acción: por un lado el contexto político y socioeconómico que envuelve a los ciudadanos los obliga a un nuevo tipo de relación con el Estado, definiéndose así una esfera micro que responde a las identidades e intereses particulares de cada grupo social o de cada actor. Por otro lado dichos actores desde sus propios contextos ejecutan luchas por la instauración de un nuevo sistema político y de prácticas económicas, sociales y culturales en sus estados nacionales.

Hasta aquí se ha examinado el concepto de movimiento social más allá de su uso común y restringido referido a procesos organizativos, acciones colectivas de protestas o simples revueltas populares. Es preferible comprender al MS en su relación con el cambio social, tal como lo expresa Jorge León Trujillo: la expresión de cómo se da el conflicto social en las sociedades modernas, y como ese conflicto caracterizado por la tensión entre actores antagónicos, que sin ser revolucionarios, termina impactando a la dinámica social. Pero si bien el concepto de MS se relaciona con los factores de cambio social, éste ha tenido distintas interpretaciones dependiendo de dónde se lea. Una manera es cómo lo interpretan los europeos y otras los norteamericanos. Desentrañar este punto lleva a la segunda parte de este ensayo: el tema de los enfoques o de las escuelas de análisis.

\section{PRINCIPALES ENFOQUES DE ANÁLISIS DE LOS MOVIMIENTOS SOCIALES}

El interés de estudiar los movimientos sociales desde la sociología se fortaleció desde la década de 1960. La intensidad de fenómenos de movilización de masas y el surgimiento de acciones colectivas novedosas por actores sociales que antes no habían irrumpido en la esfera pública llamó la atención de centenares de investigadores. Estas nuevas formas de acción exigieron nuevos modelos analíticos que explicaran las tendencias.

De manera particular surgen dos tendencias diferenciadas sobre el análisis de los MS. En los Estados Unidos los investigadores centran su análisis de los movimientos sociales desde un enfoque estratégico, privilegiando las formas de organización, los recursos y el interés de los actores en el momento de su movilización. A este marco se le denominó la teoría de movilización de recursos (TMR). En Europa, en tanto, la preocupación conceptual se centró en los factores estructurales y de identidad que motivaban la acción del MS. Esta situación permitió la creación de la teoría de los nuevos movimientos sociales (TNM).

El enfoque americano de los movimientos sociales. La estrategia, los recursos y los actores. Lo central en el enfoque americano sobre los MS es la teoría de la movilización de recursos (TMR). Esta hace énfasis en el análisis de los recursos, la organización y las oportunidades de los actores como medios que posibilitan la movi- 
lización y la consecución de objetivos.

El paradigma TMR se inspira en la clásica teoría de la acción racional. Esta escuela entiende los MS como una acción colectiva desarrollada por actores racionales que tienen un cálculo estratégico al momento de movilizarse. Se trata de un postulado originario de Olson (1963) quien entendió el cálculo estratégico como la acción de individuos racionalmente movidos por el interés propio. Es decir, la acción colectiva sólo sería posible cuando en el contexto social existan incentivos claros y concretos para los individuos o actores racionales. De esta manera, la acción colectiva es determinada por el interés. De allí que la TMR valora un MS en tanto los individuos de manera estratégica se mueven calculando costos y beneficios de su participación en la acción colectiva. En términos de Munck, "la TMR presenta así al MS como la búsqueda del fundamento a nivel micro de un fenómeno macro" (1993:20).

Desde Olson lo clave para entender porqué un actor o individuo participa o se moviliza en una acción es el tipo de incentivo que selectivamente lo mueva. Pero esta conclusión presenta un problema al suponer que para que exista la participación se requiera del incentivo, por tanto si no hay incentivo no habría acción, o al menos la acción sería imposible, y si es posible sería irracional. La respuesta entonces a este interrogante, conduce a comprender porqué verdaderamente se produce la acción colectiva; o bien tratar de entender que para darse un MS se requiere una racionalidad estratégica e instrumental, como uno de sus fundamentos. De alli que se desprendan varias observaciones al modelo de la TMR. La primera y quizá la más importante tiene que ver con que el enfoque no es monolítico, sino que por el contrario, a partir de la base antes expuesta se desprenden varias maneras de resolver la cuestión del origen y la formación de los movimientos sociales.

De modo que dentro de la TMR hay varias tendencias. En primer lugar sobresale Sydney Tarrow (1998) quien considera que el análisis de los MS debería alejarse de las formulaciones inspiradas en Olson que ubican al individuo y su interés como el eje central de la acción colectiva. Para Tarrow toda acción colectiva de un MS encarna un problema social antes que individual. Esto por cuanto la cuestión no es superar problemas personales, sino de conseguir que los indivi- duos, que forman diferentes organizaciones, actúen de manera ininterrumpida por un fin común. De modo que el interés analítico de la acción colectiva tiene que ver con la forma como los actores se organizan, cómo usan los recursos para solucionar sus problemas.

Otra lectura sobre los MS es la que hace Jenkins (1983) y Cohen (1985). Ellos para evitar confusiones sobre la TMR precisan presupuestos que explican el contenido de un MS. Estos básicamente tienen que ver con:

a) Los MS realizan una acción colectiva de carácter racional con base en cálculos estratégicos de costo y beneficio que formulan sus actores;

b) La acción colectiva puede ser institucional o no institucional. Pero ambas se inscriben en conflictos de intereses formados dentro de las relaciones de poder institucionalizadas.

c) Los MS más bien dependen de cambios en la disponibilidad de los recursos, de la organización del grupo y del marco de oportunidades existentes que garantizan la acción colectiva. Y justamente son las organizaciones formales y centralizadas las que demuestran más eficacia a la hora de movilizar recursos y por tanto son las que garantizan el éxito.

Otra visión es la de Anthony Oberschall (1973), quien considera que un MS se compone no por individuos sino por organizaciones o comunidades donde interactúa el individuo. Se trata de redes sociales o grupos solidarios con el que el individuo se identifica. Pero para que estos individuos se movilicen deben existir estímulos, recompensas o sanciones. Aquí el actor individuo es capaz de movilizarse en tanto en el grupo se alude a procesos de interés común, tal como descontentos o motivaciones compartidas. En este escenario el factor racional es evidente, el grupo sopesa recompensas, sanciones, costos y beneficios de toda acción colectiva que represente para ellos.

Ya en 1977 John D. Mac Carthy y Mayer N. Zald, siguiendo a Oberschall, privilegian el factor organización en la naturaleza de los MS. Para ellos un MS son estructuras organizacionales de preferencias dirigidas hacia el cambio social. Así todo MS posee una organización que funciona de forma compleja o formal bajo unos objetivos con el cual los actores se identifican y se diferencian. Visto así las cosas, las organizaciones son las que concretan un MS. El objetivo máximo de estas organizaciones es mantener su continuidad, pues 


\section{Bioetnia Volumen 5 No 1 (enero-junio), 2008}

de ellas depende la existencia del MS. Pero se trata de organizaciones que no son rígidas, más bien su estructura depende de factores diversos que rodean su ambiente; de allí que ellas son susceptibles a cambios e incluso sus objetivos pueden llegar a adaptarse a las circunstancias.

Un aspecto diferente en la escuela norteamericana de los MS es el enfoque del proceso político o "Estructura de las oportunidades políticas». Este enfoque de la TMR se aleja de aquel que hace énfasis en la organización y los recursos. Aquí lo clave es la importancia del entorno institucional y político en el que se produce la acción colectiva. Se trata de comprender cómo el contexto político, o nivel macro que explica Fals Borda (1989), influye en la formación, impacto y supervivencia de los MS. Este postulado que analiza la manera como el contexto político determina la acción colectiva es sostenido por autores como Singer, Gamsom, Tilly y Cloward (Rubio, 2004).

Es precisamente Singer (1973) quien introduce el concepto de "estructura de oportunidades políticas" la cual se comprende como la función de probabilidad que tiene los grupos de acceder al poder y manipular el sistema politico. Gamson (1975) por su parte, ve la importancia que tiene para los MS la realización de alianzas políticas e institucionales como medio para acceder al sistema y establecer el éxito de sus acciones colectivas. Por su parte Piven y Cloward (1977) privilegian los factores de inestabilidad electoral o política como una apertura del sistema que termina siendo favorable a las reivindicaciones de grupos movilizados (Rubio, 2004).

Pero es Tilly (2004) quien profundiza en el enfoque al entender la acción colectiva como un proceso conflictivo basado en la interacción entre actores desafiantes y actores institucionales. De manera que la acción colectiva depende de la manera como se da la interacción antagónica entre los dos actores, donde uno de ellos ejerce el poder por medio del gobierno y el otro como el que apela a subvertir ese poder. Por ello, un MS depende de la interacción entre instituciones y actores sociales.

Por su parte Doug Mc Adam (1997) señalan otros aspectos macropolíticos de los MS los cuales inciden en su formación y evolución: las estructuras de oportunidades politicas, concepto clave en los planteamientos políticos de la acción colectiva y de los MS. Desde Tarrow "son las dimensiones consistentes del entorno político que propor- cionan incentivos para la acción colectiva al influir sobre las expectativas de éxito o fracaso de la gente" (1998: 76-77). Es decir, Tarrow hace referencia a las oportunidades de acción, las cuales dependen del grado de cierre o apertura del sistema político con respecto a presiones no institucionalizadas. La acción de oportunidad depende de la estabilidad del sistema institucional del Estado, pero también de factores menos estables, las crisis políticas y las situaciones de enfrentamientos en la arena política, la ausencia de represión, la imposición de agravios repentinos a la población, la expansión del estado de bienestar o la politización de la vida privada. Desde Tarrow entender las oportunidades políticas para la acción es un factor clave para comprender cuando es necesario emprender la acción de movilización. De la correcta calibración de este factor, de la precisión del cuándo actuar, es lo que permite generar una acción política. Identificar esos momentos es lo que se denomina los "ciclos de protesta" de un MS.

Nuevos movimientos sociales o paradigma de la identidad. Mientras en los Estados Unidos el análisis de los MS se centra en los elementos microestructurales (organización, recursos, oportunidades políticas), en el proceso político y en la acción colectiva los cuales facilitan la movilización, en Europa el enfoque analítico se centra en aspectos macroestructurales, en especial aquellos que tienen que ver con el cambio social y los procesos de construcción de identidades colectivas. Estos dos factores son los que en últimas motivan al actor para emprender la acción, más allá de los cálculos racionales de costos y beneficios defendidos por la TMR.

La teoría de los nuevos movimientos sociales (NMS) no es un complejo unitario. Más bien hay enfoques diversos. Sin embargo, hay elementos claves compartidos. Uno de ellos tiene que ver con el entendimiento de que los MS son producto de los cambios estructurales propios de las sociedades modernas. Estos cambios se dan básicamente en el campo de la economía, de la tecnología, en los órdenes políticos globales y en el escenario de la cultura. Tales transformaciones estructurales generan el ambiente propicio para el nacimiento de nuevos movimientos sociales, los cuales son caracterizados según los actores, los valores, los objetivos y las formas de acción.

Alain Touraine y Alberto Melucci son los dos principales exponentes de los enfoques de la 
teoría de los NMS. Touraine propone el paradigma de la "Sociología de la Acción" para plantear que los MS no se deben necesariamente a la existencia de un conflicto social coyuntural, en cambio son resultados de un conflicto central gestado históricamente por grupos sociales antagónicos (Touraine: 1985:772). Para este autor dentro de la variedad de expresiones de los MS, por lo menos hay dos muy característicos:

a) los movimientos históricos, que se producen por acciones organizadas para controlar el paso de una sociedad a otra;

b) los movimientos culturales, orientados a la transformación de valores.

Desde el enfoque de Touraine, los NMS son producto de las sociedades transformadas. Ellos sintetizan los conflictos que históricamente se han dado entre grupos sociales que detentan el poder y entre quienes no lo detectan. Además, tales MS desafian los valores culturales tradicionales, como por ejemplo el papel de la mujer en la sociedad, o el tema del racismo y la discriminación. Se trata de movimientos que en las sociedades modernas se extienden a todos los aspectos de la vida cultural, social y politica, son formas de vida colectivas con una fuerte identidad de grupo, pero que les corresponden competir con el individualismo muy característico de las sociedades modernas.

Por su parte Melucci (1989) destaca la construcción de identidades colectivas como motores que impulsan a los MS. Igual que Touraine, este autor considera que los NMS son producto los cambios que se dan en las sociedades posindustriales en su paso a una sociedad más compleja. Pero el rasgo distintivo de Melucci es que los MS implican cuestiones que afectan la producción cultural de las sociedades. Es decir, los NMS luchan por objetivos simbólicos y culturales, luchan por una orientación diferente y una significación distinta de la acción social. Se trata de desafiar la lógica que gobierna la producción y la apropiación de los recursos sociales. En esta búsqueda, los NMS se presentan como creadores de códigos culturales alternativos a los dominantes (Melucci: 1989:29).

Para Melucci un movimiento social y sus acciones colectivas no son unidades fijas, o datos empíricos determinados por actos de protestas. Ellos más bien corresponden a construcciones sociales que revelan un punto de partida para la transformación social (1994: 158). De alli que las acciones colectivas de los MS sean más bien el resultado de intenciones, recursos y limites construidos mediante relaciones sociales desarrolladas en un sistema de oportunidades y obligaciones.

Para Melucci si un NMS se enfrenta al sistema por cuestiones simbólicas y culturales, el término MS resultaría inadecuado. De allí que propone el concepto de "áreas de movimiento", pues es alli donde se configura la identidad colectiva. Estas áreas están estructuradas en redes sociales, compuestas a su vez por múltiples organizaciones o grupos dispersos pero conectados a manera de circuitos a través de intercambios sociales. Se trata de redes que están sumergidas en la vida cotidiana y funcionan a manera de laboratorios culturales (1989:60). En estas áreas o redes es donde se concreta la identidad colectiva, donde se forjan los vínculos para la acción, donde se dan los fundamentos de la movilización. Allí los individuos comparten la experiencia de crear nuevos modelos culturales, nuevas formas de relación que les proporciona nuevas formas de comprender el mundo. Desde Melucci las redes sociales que componen los NMS presentan una novedad en su forma de organización. Se trata de estructuras organizativas cuyo fin son ellos mismos, donde no habría mayor objetivo que el de producir mensajes, desafíos simbólicos a los códigos dominantes.

Categorias metodológicas. Hasta aqui hemos comprendido a los movimientos sociales como expresiones históricas de conflictos que actores antagónicos en una sociedad luchan por el cambio. Asimismo se han esbozados las dos principales corrientes de análisis de los MS, donde sobresale, por un lado, la norteamericana con la teoría de la movilización de recursos, mientras que en otra orilla, se destacan los autores europeos con posturas divergentes pero que descansan en lo que denomina la teoría de los nuevos movimientos sociales. Ahora se abordará un esbozo metodológico que desde los postulados anteriores permita acercarnos a un futuro estudio sobre la naturaleza del MS afrodescendiente.

Gerardo Munck (1993) en un intento apretado por precisar puntos de encuentro entre la TMR y la NMS propone 3 aspectos o categorias centrales para un análisis de los MS. En primer lugar, si un movimiento social se comprende como un "tipo de acción colectiva orientada hacia el cambio impulsada por un grupo de personas encabezadas de 


\section{Bioetnia Volumen 5 № 1 (enero-junio), 2008}

manera no jerárquica por un actor social» (Munck 1993:24), un MS debe por lo menos tener tres características:

a) actores, los cuales atañen a la fundación del movimiento,

b) una coordinación, la cual busca poner de acuerdo en algo a dichos actores, y

c) la estrategia politica, la cual se liga hacia la orientación del cambio que propone dicho MS.

Estos tres elementos, entre otros, los consideramos claves y necesario si deseamos una comprensión más sociológica de lo que podría entenderse como "movimiento social afrodescendiente".

a) La formación de los actores. Los actores constituyen el punto inicial de un MS. Ellos son los que impulsan la organización de una masa descentralizada la cual constituye la base del MS. Tarrow (1994) se ocupa de entender la dinámica de los actores y los denomina "organizadores del movimiento social". Es decir, la gente que se atreve a movilizarse o a movilizar. De igual manera Touraine (1985) considera estratégico el papel de estos agentes y los determina como centrales en la generación de la acción colectiva. Viendo las cosa de esta manera, la propuesta es analizar el fenómeno de un MS centrando el primer interés en cómo nacen estos actores, qué los motiva, en qué contexto se desenvuelven, qué factores favorecen su emergencia.

Por lo general, de acuerdo con Munck, los actores sociales emergen en medio de crisis sociales de carácter estructural. Ellos pertenecen o son sensibles de aquellos sectores subalternos que se sienten víctimas de la dominación que otro actor ejerce sobre el contexto. Se trata de agentes que comprenden el conflicto en que están inmersos y proponen el establecimiento de un nuevo orden social.

Estos actores se forman gracias a que poseen una visión de que las cosas deben tener un orden diferente. Ellos catalizan esta visión, la cual de forma latente es compartida por el grupo social. De modo que la comunión de una visión común diferente se convierte en una llama que enciende la movilización social. Pero para que esta llama tenga vida es necesario que exista la identidad colectiva.

b) La coordinación social. Pero la emergencia de los actores sociales no es garantía del desarrollo de un MS. Pues una vez encendida la llama de la movilización, el desafio ahora es cómo mantenerla viva, cómo orientarla hacia el cambio. Incluso Tarrow (1994) plantea el problema de forma más compleja: ¿Cómo pueden los actores fundadores controlar a sus seguidores, cómo pueden darle coherencia a sus acciones? Para el autor, los actores fundadores encaran el problema de la coordinación social gracias a la utilización adecuada de los medios, o de los recursos existentes, tales como los repertorios de acción, la estructura de las oportunidades políticas, o bien utilizando estratégicamente las disputas entre redes sociales y los marcos culturales en que los actores tienen a su disposición.

Concretamente, siguiendo a Tarrow, los fundadores del MS logran éxito conduciendo a sus bases sociales gracias al aprovechamiento de las estructuras de las oportunidades politicas. Aunque a criterio de Munck son los repertorios de disputa, las redes sociales y los marcos culturales que al ligarse con las habilidades de los actores fundadores permiten el éxito de la coordinación. Mientras que las estructuras de las oportunidades politicas dependen más de la estrategia política que de la coordinación.

c) La orientación hacia el cambio. Si bien los actores fundadores logran la coordinación de sus bases sociales para la movilización, la pregunta ahora es cómo orientar el MS hacia el cambio. En primer lugar hay que recalcar que los MS son más que organizaciones. Los MS al proponer el cambio se enfrentan a un problema de estrategia politica, es decir, de cómo relacionarse con las instituciones que confrontan, cómo entran en contacto con ellas y con otros procesos de movilización. Se trata de un problema que pone a prueba la capacidad y la habilidad de los actores fundadores para relacionarse estratégicamente con sus adversarios. Es decir, un problema de cómo determinar su estrategia.

Para Munck los MS emprenden su estrategia política hacia el cambio tanto desde sus conexiones de identidad colectiva como desde las estrategias del actor. O sea, tanto de la constitución estructural de la identidad de un MS como de la posibilidad de su acción estratégica. Estos dos elementos nos aproximan a una definición más precisa de un MS que se podría entender como un tipo de acción colectiva orientada al cambio social (Tarrow 1994, Melucci 1989).

La orientación hacia el cambio obliga a los actores del MS a actuar como actores estratégicos, a moverse en relación con su medio, a 
orientar sus fines de acuerdo con las circunstancias, considerando siempre los factores de riesgo, de oportunidades y de amenazas.

Pero la orientación sólo es efectiva si el actor es conciente que no debe jamás perder su identidad, que no debe perder su naturaleza en el ajedrez de la negociación estratégica. De allí que la orientación hacia el cambio debe conjugar tanto la acción estratégica (TMR) como la radicalidad de su identidad colectiva (TNM). Pero no siempre esta conexión funciona. Muchas veces se sobrepone la estrategia a la identidad. Desde Roberto Unger (1987:396) estas cosas ocurren cuando los actores inspirados en la política buscan los objetivos sin importar los medios. Y la manera más concreta de observar este fenómeno es cuando el MS al entrar a un entorno político que busca transformar, más bien el entorno termina transformándolo a él.

Igualmente, la relación estrategia-identidad también se rompe cuando las condiciones estratégica son descuidadas a nombre del privilegio esencialista de la identidad. Esto ocurre cuando los MS calculan que la estrategia los puede dominar a expensa de la visión que los motiva. De modo que terminan refugiándose en actividades puramente expresivas de su identidad, muchas veces sin contenido político. Y el desafio de transformar la realidad termina ignorado. La estrategia política en sí se debilita y los objetivos de cambio son impedidos.

En suma, el manejo exitoso de la estrategia politica hacia el cambio depende de la habilidad de los organizadores de un MS para confrontar el peligroso problema de los medios y de los fines. Y aunque se pueden impulsar estrategias que sean compatibles con la identidad y con las metas, la clave es mantener los mecanismos entre lo negociable y lo no negociable. Se trata de proteger los objetivos estructurales transformadores y eliminar las acciones puramente instrumentales. E1 actor debería actuar estratégicamente en medio de una racionalidad puramente instrumental.

\section{CONCLUSIONES}

Las movilizaciones y acciones colectivas de los afrodescendientes en los últimos 20 años es un fenómeno digno de estudio por parte de las ciencias sociales. Se trata de un interesante proceso que con espectacularidad se ha dado no solo en Colombia sino en toda la región. Sin embargo, para una correcta comprensión analítica de la dimensión real del movimiento social afrodescendiente, es importante disponer de un marco referencial suficiente.

En este ensayo, más que un análisis sociológico del movimiento afrodescendiente, se ha intentado realizar una breve reseña de las teorias más relevantes del movimiento social. En un intento de definición de lo que puede ser un MS partimos de los argumentos de Jorge León (1997) quien asocia a los MS con los fenómenos de cambio que las sociedades modernas experimentan en un determinado tiempo histórico. Así, León considera que un MS "no es una organización ni un programa definido. Es la convergencia de ideas, acciones y personas que se encaminan hacia transformaciones de sus situaciones o instituciones definidas. (1997:30). Este concepto abarca variadas formas de acción colectivas adaptadas a ciertas características del cambio social y a características de la acción (revueltas, protestas) o características de los actores. En términos generales un MS puede expresarse o no en acciones colectivas, puede tener varios actores (organizaciones, instituciones, sectores sociales, ONGs), e integrar diversos catalizadores (organizaciones no gubernamentales, iglesia, entidades transnacionales). En suma, un MS es una categoría abarcadora de un fenómeno que identifica un proceso social por el cual una sociedad cambia uno o varios aspectos de su vida o de sus instituciones" (1997: 30).

Ahora bien, en este ensayo se ha intentado un esbozo de los principales enfoques teóricos de los movimientos sociales. Estos básicamente son dos: el enfoque norteamericano y el enfoque europeo. Estos enfoques se consolidan en las décadas de 1960 y 1970 cuando las nuevas perspectivas se alimentan no sólo de la observación, sino también de la intervención de los investigadores. De acuerdo con Cohen (1985) en este escenario renovador de las ciencias sociales se radicalizan dos formas de interpretación de los MS. En los Estados Unidos se privilegia el enfoque estratégico de los actores y en Europa se distingue el carácter identitario de los actores en la acción. En Estados Unidos se consolida la teoría de la movilización de recursos (TMR) con los trabajos de Anthony Oberschall, John McCarthy y Mayer Zald. Más adelante surge Charles Tilly quien profundiza y renueva el enfoque. Por su parte, en Europa se consolida el paradigma de los "nuevos 


\section{Bioetnia Volumen 5 No 1 (enero-junio), 2008}

movimientos sociales" con Alain Touraine, Claus Offe, Francisco Alberoni y Alberto Melucci. Estos autores centran su atención en los conflictos y cambios culturales y macroestructurales de las sociedades modernas, los cuales dan lugar a la formación de nuevas identidades que se expresan en lo que se denomina "nuevos movimientos sociales".

Luego de hacer esta revisión conceptual, las notas concluyentes de este ensayo permiten comprender a los MS como la expresión de actores que se representan de manera alternativa a las tradicionales formas del quehacer politico, encarnando lo que G. Munck denomina "una nueva politica de movilización"(1993:19). Es decir, los MS aparecen como procesos alternativos irreverentes y contradictores de los partidos políticos, de los sindicatos y de las maquinarias politicas populistas y clientelares. Además, se reivindican como descontaminantes o refrescantes a las tendencias politicas alimentadas por la izquierda radical.

En términos de Orlando Fals Borda, existe una convergencia de movimientos sociales criticos y radicales cuyas reivindicaciones parecen traspasar la frontera de la tradicional demanda de mejores servicios por parte del Estado. Hoy nos encontramos frente a movimientos sociales y actores políticos de varios tipos: por la restauración democrática, por los derechos humanos, por la diversidad sexual, por el derecho internacional humanitario, por los cristianos protestantes, por los católicos de base, por las mujeres, jóvenes y los movimientos étnicos.

En América Latina a finales de la década de 1970, junto con los movimientos indigenas y campesinos, emergen los movimientos de las comunidades negras, que desarrollan acciones colectivas en torno a la reafirmación étnica y en contra de la discriminación racial. Los afrodescendientes en la región se movilizan en medio de un complejo proceso de construcción étnica, tejido desde un horizonte ancestral, histórico y político. Dicho fenómeno se ha venido configurando en medio de formas de reconstrucción y revaloración de su identidad cultural que, además, les ha permitido autodefinirse como una "etnia" o bien un grupo humano que comparte una cultura y cuyos miembros están unidos por una conciencia de identidad común establecida históricamente.

\section{LITERATURACITADA}

Agudelo L.E. 2006. Poblaciones negras y politica en el Pacífico Colombiano: paradojas de una inclusión antigua. Bogotá: Icanh.

Antón-Sánchez J. 2004. Organizaciones de la sociedad civil afroecuatoriana. Documento Mimeografiado. Quito: Banco Interamericano de Desarrollo (BID).

Cohen, J. 1985. Strategy or identity: New theorical paradigms and contemporary social movements". Social Res. 52 (4).

De la Torre C. 2003. Movimientos étnicos y cultura politica en Ecuador. Rev FLACSO. 15.

Escobar A. 1997. Política cultural y biodiversidad: Estado, capital y movimientos sociales en el Pacífico colombiano. En: Restrepo y Uribe (eds). Antropología en la modernidad. Bogotá: ICAN.

Fals Borda O. 1989. Movimientos sociales y poder politico. Rev Democracia. Presentado en el VII Congreso Nacional de Sociología. Barranquilla.

Handelsman M. 2001. Lo afro y la plurinacional. Quito. Abya Yala.

Oberschall A. 1973. Social conflic and social movements. New York: Prentice Hall, Englewood Cliffs.

León-Trujillo L. 1997. Entre la propuesta y el corporativismo. En: ICONOS. Rev FLACSO. 2.

Mac Adam, D., Mac Carthy, J. D., y Zald, M. N. 1973. Social movements. En: Smelser, N.J. (ed). Handbook of sociology. Beverly Hills, London: Sage.

Mac Adam, D., Mac Carthy, J. D., y Zald, M. N. 1996. Comparative perspectives on social movements: political opportunities, mobilizing structures and cultural fragmings. Cambridge, New York: Cambrige University Press.

Melucci A. 1994. Asumir un compromiso: identidad y movilización en los movimientos sociales. Madrid: Zona Abierta No 69 .

Melucci, A 1989. Nomads of the present: Social movements and individual needs in contemporary society. London: Hutchinson Radius.

Mouriaux R. y Beroud S. 2000. Para una definición del concepto de "movimiento social". OSAL junio 2000.

Munck G. L. 1993. Algunos problemas conceptuales en el estudio de los movimientos sociales. Rev Mex Sociol.

Neveu E. 2000. Sociología de los movimientos sociales. Quito: Abya Yala.

Rubio-García A. 2004. Perspectivas teóricas en el estudio de los movimientos sociales. Circunstancia. 1 (3).

Tarrow S. 1998. El Poder en movimiento: Los movimientos sociales, la acción colectiva y la politica. Madrid: Alianza Editorial.

Tilly C. 2000. La desigualdad persistente. Buenos Aires: Manantial.

Tilly C. 2004. ¿De dónde vienen los derechos? Sociología 19 (55).

Tilly C. Acción colectiva. Rev Apuntes de Investigación del CECYP VI (6).

Touraine A. 1985. El regreso del actor. Buenos Aires: Editorial Universitaria de Buenos Aires.

Unger R. 1987. False necessity. Anty-necessitarian social theory in the service of radical democracy. New York: Cambridge University Press. 